\title{
Surface plasmon on aluminum concentric rings arranged in a long-range periodic structure
}

\author{
Yi-Tsung Chang, Dah-Ching Tzuang, Yi-Ting Wu, Chi-Feng Chan, Yi-Han Ye, \\ Ting-Hsiang Hung, Yu-Fan Chen, and Si-Chen Lee ${ }^{\text {a) }}$ \\ Department of Electrical Engineering, Graduate Institute of Electronics Engineering, National Taiwan \\ University, Taipei, Taiwan 10617, Republic of China
}

(Received 4 April 2008; accepted 2 June 2008; published online 25 June 2008)

\begin{abstract}
The enhanced optical transmission through the annular aluminum metal with different number of concentric rings arranged in a long-range periodic structure on the silicon substrate is investigated. The measured transmission spectra demonstrate that the surface plasmon modes depend sensitively on the structural parameters of the metallic rings. The degenerate $(1,0),(1,1)$, and $(2,1) \mathrm{Al} / \mathrm{Si}$ surface plasmon modes are determined by the long periodicity of the structure. The localized modes in cavities with different length can be observed in this structure as well. These phenomena suggest that both surface plasmons and localized cavity modes are excited within super unit cell.

(C) 2008 American Institute of Physics. [DOI: 10.1063/1.2949743]
\end{abstract}

Recently, different configurations of subwavelength holes $^{1,2}$ on metallic film have been proposed and studied experimentally which is of great interest for many applications such as magneto-optic data storage, ${ }^{3}$ quantum dot infrared photodetector, ${ }^{4}$ and plasmonic thermal emitter. ${ }^{5}$ The transmission through metal film with a metallic coaxial apertures consisting of a concentric arrangement of a solid disk and a surrounding ring have been investigated. ${ }^{6,7}$ The transmission spectra exhibit clear optical resonance in the visible to infrared spectral range. It demonstrates that the energies of the plasmon modes depend sensitively on the structural parameters of the disk and ring. As the distance between ring and disk increases, the effective coupling between the localized mode and the surface plasmon (SP) weakens that causes the transmission peak to redshift. These annular aperture structures were demonstrated to dramatically increase the light transmission as compared to circular or rectangular apertures. ${ }^{7,8}$ The degenerate $(1,0)$ metal/dielectric SP modes and harmonic peaks were not clearly explained. This letter presents the studies of an aluminum ( $\mathrm{Al}$ ) film perforated with microcells ${ }^{2}$ arranged in long-range periodic structure. The individual microcell comprises a number of concentric rings, i.e., one to eight rings. The sizes and lattice type of the microcells are varied to examine their effect on the extraordinary transmission spectra and the dispersion relation of SP polaritons (SPPs). ${ }^{9}$

The sample consists of a Si substrate and a top planar Al film perforated with microcells arranged in a long-range periodic structure, the side and top views are displayed in Figs. 1(a)-1(c), respectively. Figures 1(b) and 1(c) display the individual microcell comprises two and four metal rings, respectively. The metal films were prepared by depositing 200-nm-thick Al on a doubly polished silicon wafer, and the photoresist was spun onto Al films for lithography. Following pattern transfer, an annular metal structure was etched. The diameter of central hole in the concentric rings is $2 \mu \mathrm{m}$. The gap between concentric metal ring and the metal ring width are both $1 \mu \mathrm{m}$ in the microcells, respectively. The superperiodicity of the microcells is $p$ in the larger periodic

\footnotetext{
${ }^{a)}$ Electronic mail: sclee@cc.ee.ntu.edu.tw.
}

lattice, where $p=13$ and $33 \mu \mathrm{m}$ in Figs. 1(b) and 1(c), respectively. The sample is defined to lie in the $(x, y)$ plane, and rotated about the $y$ axis with various $\Omega$. The unpolarized light is incident in the $z$ direction, allowing the dispersion relation in the $x$ direction to be examined. The transmission spectra as displayed in Figs. 2 and 3 were measured and analyzed using a Bruker IFS 66V/S Fourier-transform infrared spectrometer.

For a two-dimensional periodic hexagonal holes array, at normal incidence $\left(k_{x}=0\right)$, SP modes appeared at wavelength $\lambda_{\mathrm{SP}}$

$$
\lambda_{\mathrm{SP}}=a\left[\frac{4}{3}\left(m^{2}+m n+n^{2}\right)\right]^{-1 / 2}\left(\frac{\varepsilon_{d} \varepsilon_{m}}{\varepsilon_{d}+\varepsilon_{m}}\right)^{1 / 2} .
$$

For a square holes array, the transmission wavelength $\lambda_{\mathrm{SP}}$ is given by

$$
\lambda_{\mathrm{SP}}=\frac{a}{\left(m^{2}+n^{2}\right)^{1 / 2}}\left(\frac{\varepsilon_{d} \varepsilon_{m}}{\varepsilon_{d}+\varepsilon_{m}}\right)^{1 / 2},
$$

where $a$ is the periodicity of the hole array, $\varepsilon_{d}$ and $\varepsilon_{m}$ are the dielectric constants of the dielectric material and metal, respectively, $m$, and $n$ are integers that correspond to the par-

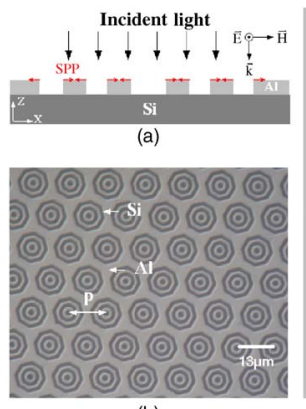

(b)

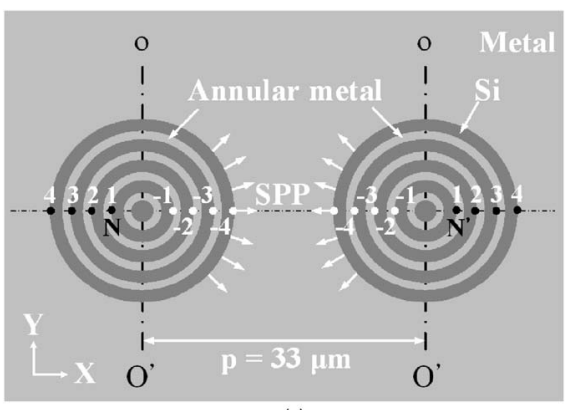

(c)
FIG. 1. (Color online) The structure consists of a concentric arrangement of metallic rings on $\mathrm{Al}$ film. The diameter of central hole is $2 \mu \mathrm{m}$, the distance between metallic rings (gap) is $1 \mu \mathrm{m}$, and the width of metallic ring is also $1 \mu \mathrm{m}$. The (a) side view and (b) top view of the hexagonal lattice with lattice constant $p=13 \mu \mathrm{m}$, each microcell comprises two metallic rings, and (c) the top view of squared lattice with lattice constant $p=33 \mu \mathrm{m}$, each microcell comprises four metallic rings. 


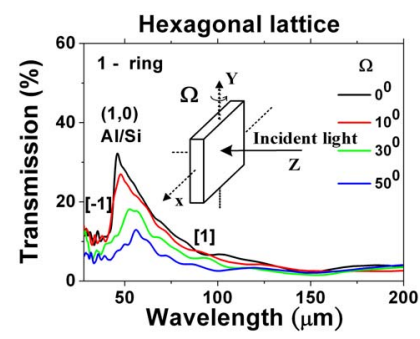

(a)

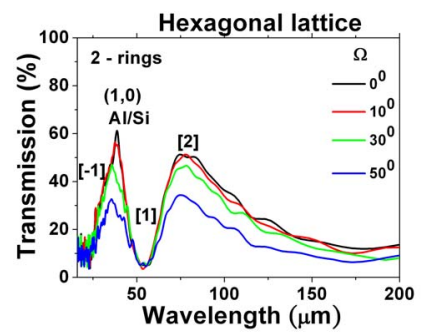

(b)

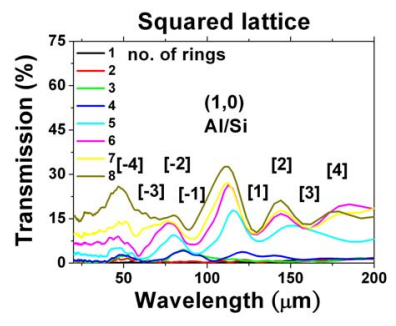

(c)

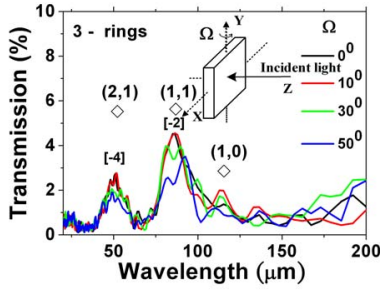

(a)

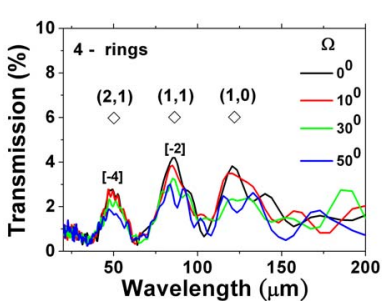

(b)

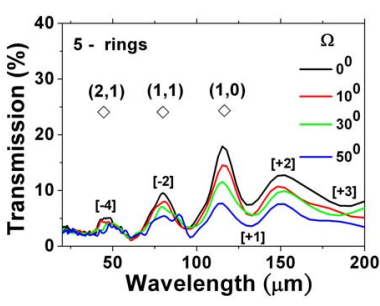

(c)

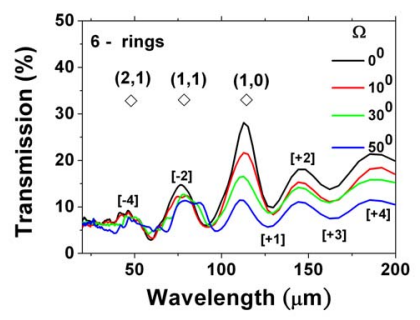

(d)

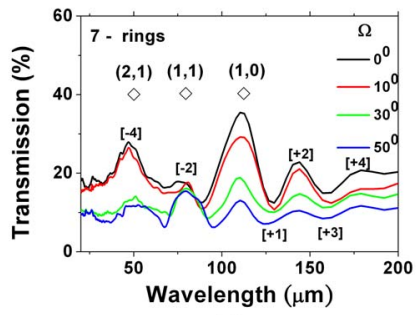

(e)

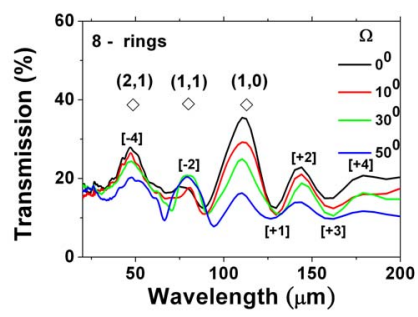

(f) ticular order of the SPP. The dielectric constant of air $\left(\varepsilon_{\text {air }}\right.$ $=1$ ) and the real part of the dielectric constant of the dielectric material (Si wafer, $\left.\varepsilon_{\mathrm{Si}}=11.9\right)$ differ. ${ }^{2,9,10}$

Figures 2(a)-2(c) display the transmission spectra of the microcell arrays at various $\Omega$. The samples are arranged in hexagonal arrays in Figs. 2(a) and 2(b) and squared array in Fig. 2(c). The microcell comprises one metallic ring with lattice constant $p=15 \mu \mathrm{m}$ in Fig. 2(a), two metallic rings with $p=13 \mu \mathrm{m}$ in Fig. 2(b), and different number of rings (one to eight) with $p=33 \mu \mathrm{m}$ in Fig. 2(c). According to Eq. (1), the transmission spectra shown in Fig. 2 should display only one $(1,0) \mathrm{Al} / \mathrm{Si} \mathrm{SP}$ mode at normal incidence of radiation at a theoretical wavelength of approximately $45 \mu \mathrm{m}$, the measured value is $45.8 \mu \mathrm{m}$. The intensity of SPP is increased comparing to traditional circular or squared periodic holes array, ${ }^{2,45}$ it is attributed to interaction of SPP within annular cavity. In Fig. 2(b), the lattice constant of the hexagonal lattice with two metallic rings in each microcell exhibit two peaks, the $(1,0) \mathrm{Al} / \mathrm{Si}$ SP peak can be calculated from Eq. (1) to locate at a wavelength of approximately $38.8 \mu \mathrm{m}$ (the measured values are $39 \mu \mathrm{m}$ ). The other peak is seen at a wavelength of $71.3 \mu \mathrm{m}$, the theoretical value is $69 \mu \mathrm{m}$ using a longer lattice constant $a=23 \mu \mathrm{m}$. This suggests that the corresponding SPP resonates in a longer cavity.

As defined in Fig. 1(c), a line through the centers of the rings connecting the inside of the $(n+1)$ th ring of two neighboring microcells with two edges $N, N^{\prime}$ is defined as $[ \pm n] N N^{\prime}$. Positive (negative) sign $+(-)$ means the length of cavity is larger (smaller) than the lattice constant. For sample in Fig. 2(b), $[+2] N N^{\prime}$ means line $2 \overline{2}=23 \mu \mathrm{m}$. This mode is defined as Fabry-Pérot (FP) mode since its peak position is independent on incident angle which indicates a zero group velocity. For $[+1] N N^{\prime}$ cavity with length $19 \mu \mathrm{m}$, the transmission spectra shows a minimum at theoretical value $57 \mu \mathrm{m}$. The oscillation of transmission spectra is atvalue $57 \mu \mathrm{m}$. The oscillation of transmission spectra is at- for four metal rings and beyond.
Downloaded 04 Mar 2009 to 140.112 .113 .225 . Redistribution subject to AIP license or copyright; see http:

FIG. 3. (Color online) Transmission spectra of $\mathrm{Al} / \mathrm{Si}$ structure with the concentric rings in squared lattice. The period of the larger periodic structure $p$ is $33 \mu \mathrm{m}$, and number of annular metal rings is (a) 3, (b) 4 , (c) 5 , (d) 6 , (e) 7 , and (f) 8 .

tributed to the interference of different cavity modes with the same cavity length, as displayed in Fig. 1(c). The phenomena are discussed in detailed as follows. As normally unpolarized incident light is used for SP excitation, as shown in Figs. 1(a) and 1(c). The in-plane wave vector will come entirely from the diffraction of light resulting from the annular metal grating. The direction of the excited SPP wave vector which determines the energy propagation direction will be perpendicular to the metallic rings. Consequently, in the case of an annular metal structure, the energies will be guided toward the central hole, and SPPs are distributed on both sides of the symmetric axis $\left(O O^{\prime}\right)$ as displayed in Fig. 1(c). ${ }^{11}$ Figure 2(c) displayed the transmission spectra with one to eight metal rings in squared lattice with the same lattice constant $p$ $=33 \mu \mathrm{m}$. The SP mode of one metal ring cannot be seen, it is owing to the distance between microcells is too long. The intensity of SP modes is increased when the number of annular metal ring increases. For eight metal rings, the $(1,0)$, $(1,1)$, and $(2,1) \mathrm{SP}$ modes $\mathrm{Al} / \mathrm{Si}$ and order [ \pm 1$],[ \pm 2],[ \pm 3]$, and $[ \pm 4]$ harmonic modes corresponding to lattice constant $a=[ \pm n] N N^{\prime}$ for $n=+1(-1)$ to $+4(-4)$, respectively, were observed. According to Eq. (2), the peak positions of theoretical, experimental SPP modes, and harmonic modes are listed in Table I for samples with different number of metal rings in squared lattice. Through detailed analysis, it is discovered that the transmission peaks are determined by SPP and FP type cavity modes within microcell. The theoretical values are very close to the experimental values especially

do AIP license or copyright; see http://apl.aip.org/apl/copyright.jsp 
TABLE I. The theoretical and experimental values of the transmission peak wavelengths of the $\mathrm{Al} / \mathrm{Si}$ annular metal structure in squared lattice with lattice constant $p=33 \mu \mathrm{m}$ based on FP type resonance and SPPs in superperiodic microcell (unit: micrometers). $T$ for theoretical value and $\mathrm{M}$ for experimental value.

\begin{tabular}{|c|c|c|c|c|c|c|c|}
\hline \multirow[b]{2}{*}{ SPP mode } & & \multicolumn{6}{|c|}{ Ring No. } \\
\hline & & 3 & 4 & 5 & 6 & 7 & 8 \\
\hline Order & $T$ & \multicolumn{6}{|c|}{$176\left([+4] N N^{\prime}=51 \mu \mathrm{m}\right)$} \\
\hline$[+4]$ & $M$ & 161.1 & 173.6 & 207 & 178 & 176.8 & 177 \\
\hline Order & $T$ & \multicolumn{6}{|c|}{$162.2\left([+3] N N^{\prime}=47 \mu \mathrm{m}\right)$} \\
\hline$[+3]$ & $M$ & 148.7 & 158.4 & 187.6 & 160.6 & 161.7 & 160.5 \\
\hline Order & $T$ & \multicolumn{6}{|c|}{$148.4\left([+2] N N^{\prime}=43 \mu \mathrm{m}\right)$} \\
\hline$[+2]$ & $M$ & 136.6 & 142.3 & 150.8 & 146.5 & 144.5 & 144.4 \\
\hline Order & $T$ & \multicolumn{6}{|c|}{$131.1\left([+1] N N^{\prime}=39 \mu \mathrm{m}\right)$} \\
\hline $\begin{array}{l}{[+1]} \\
(1,0)\end{array}$ & $M$ & 128.1 & 132.4 & 132.3 & 129.2 & 128.1 & 129.7 \\
\hline $\begin{array}{l}\mathrm{Al} / \mathrm{Si} \\
\mathrm{SP}\end{array}$ & $T$ & \multicolumn{6}{|c|}{$113.9(p=33 \mu \mathrm{m})$} \\
\hline mode & $M$ & 115.7 & 120.6 & 115.6 & 113.4 & 112.7 & 112.3 \\
\hline Order & $T$ & \multicolumn{6}{|c|}{$93.2\left([-1] N N^{\prime}=27 \mu \mathrm{m}\right)$} \\
\hline $\begin{array}{l}{[-1]} \\
(1,1) \\
\mathrm{Al} / \mathrm{Si}\end{array}$ & $M$ & 110.1 & 103.5 & 93.3 & 92.3 & 91.3 & 89.3 \\
\hline $\begin{array}{l}\mathrm{SP} \\
\text { mode } \\
+[-2]\end{array}$ & $T$ & \multicolumn{6}{|c|}{$79.4\left([-2] N N^{\prime}=23 \mu \mathrm{m}\right)$} \\
\hline mode & $M$ & 85.6 & 85.4 & 79.9 & 78.2 & 78.7 & 80.7 \\
\hline Order & $T$ & \multicolumn{6}{|c|}{$65.6\left([-3] N N^{\prime}=19 \mu \mathrm{m}\right)$} \\
\hline $\begin{array}{l}{[-3]} \\
(2,1) \\
\mathrm{Al} / \mathrm{Si}\end{array}$ & $M$ & 68.6 & 64.2 & 62 & 59.4 & 57.3 & 65.9 \\
\hline $\begin{array}{l}\mathrm{SP} \\
\text { mode } \\
+[-4]\end{array}$ & $T$ & \multicolumn{6}{|c|}{$51.8\left([-4] N N^{\prime}=15 \mu \mathrm{m}\right)$} \\
\hline mode & $M$ & 50.7 & 51.2 & 50.1 & 47.2 & 50.7 & 48 \\
\hline
\end{tabular}

Figures 3(a)-3(f) displayed dispersion curves of SPPs with different number of metal rings. The $\diamond$ represents $(1,0)$, $(1,1)$, and $(2,1) \mathrm{Al} / \mathrm{Si} \mathrm{SP}$ modes. When the number of metallic rings is less than five, all three $\mathrm{Al} / \mathrm{Si} \mathrm{SP}$ modes spilt and change peak wavelengths as the rotation angle $\Omega$ increases indicating they are propagating modes. However, when the number of metallic rings is five and beyond, the $(1,0) \mathrm{Al} / \mathrm{Si} \mathrm{SP}$ becomes a localized mode and the angularindependent FP modes also appear, as shown in Figs. 3(c)-3(f). They are the localized cavity modes within microcells such as $[+1],[+2],[+3]$, and $[+4] N N^{\prime}$ harmonic modes. In Figs. 3(a) $-3(\mathrm{f})$, the $[-2]$ and $[-4] N N^{\prime}$ harmonic modes are overlapped with $(1,1)$ and $(2,1) \mathrm{Al} / \mathrm{Si} \mathrm{SP}$ modes which are angular-dependent even the number of metallic rings is five and beyond. It is believed that the harmonic modes $[-n]$ are still localized modes with weaker intensity. This phenomenon is interesting to note that when the distance between the outmost annular aperture of two neighboring microcell, i.e., $11 \mu \mathrm{m}$ when number of metal ring is five, is smaller than $\frac{1}{3}$ of the lattice constant $p(=33 \mu \mathrm{m})$, the $(1,0) \mathrm{Al} / \mathrm{Si} \mathrm{SP}$ becomes a localized mode. It is owing to the overlap of $[+n] N N^{\prime}$ modes between neighboring cavities. The even modes (peak) are found to satisfy the SP excitation condition and can be calculated by Eqs. (1) and (2). The odd modes (dips) can be explained by Rayleigh-Wood's anomaly ${ }^{10}$ and satisfy Eqs. (1) and (2), it is owing to the fact that $\varepsilon_{m} \gg \varepsilon_{d}$.

In conclusion, the experimental evidences demonstrated that the intensity of $(1,0)$ SP mode and FP modes can be enhanced by increasing the number of metallic ring. The degenerate $(1,0) \mathrm{Al} / \mathrm{Si} \mathrm{SP}$ modes is determined by the period of the lattice $p$. $[ \pm n] N N^{\prime}$ modes can be regards as localized FP modes, the $(1,0),(1,1)$, and $(2,1) \mathrm{Al} / \mathrm{Si} \mathrm{SP}$ modes can be treated as propagating modes when the number of metal rings is less than five. These observations suggest that the cavity modes within two microcells can be excited. It can be applied to various devices such as light emission diode, the plasmonic thermal emitter, wavelength selective quantum dot (ring) infrared photodetector etc.

The authors would like to thank the Center for Nano Science and Technology National Taiwan University, Taiwan, Republic of China, for partially supporting this research under Contract No. 96R0061-03.

${ }^{1}$ T. W. Ebbesen, H. J. Lezec, H. F. Ghaemi, T. THio, and P. A. Wolff, Nature (London) 391, 667 (1998).

${ }^{2}$ Y.-T. Chang, T.-H. Chuang, M.-W. Tsai, M.-J. Lai, and S.-C. Lee, Appl. Phys. Lett. 90, 253106 (2007).

${ }^{3}$ E. Betzig, J. K. Trautman, R. Wolfe, E. M. Gyorgy, P. L. Finn, M. H. Kryder, and C.-H. Chang, Appl. Phys. Lett. 61, 142 (1992).

${ }^{4}$ C.-Y. Chang, H.-Y. Chang, C.-Y. Chen, M.-W. Tsai, Y.-T. Chang, S.-C. Lee, and S.-F. Tang, Appl. Phys. Lett. 91, 163107 (2007).

${ }^{5}$ M.-W. Tsai, T.-H. Chuang, C.-Y. Meng, Y.-T. Chang, and S.-C. Lee, Appl. Phys. Lett. 89, 173116 (2006).

${ }^{6}$ Y. Poujet, J. Salvi, and F. I. Baida, Opt. Lett. 32, 2942 (2007).

${ }^{7}$ F. Hao, P. Nordlander, M. T. Burmett, and S. A. Maier, Phys. Rev. B 76, 245417 (2007).

${ }^{8}$ F. I. Baida and D. Van Labeke, Phys. Rev. B 67, 155314 (2003).

${ }^{9}$ H. F. Ghaemi, T. Thio, D. E. Groupp, T. W. Ebbesen, and H. J. Lezec, Phys. Rev. B 58, 6779 (1998).

${ }^{10}$ J. M. McMahon, J. Henzie, T. W. Odom, G. C. Schatz, and S. K. Gray, Opt. Express 15, 18119 (2007).

${ }^{11}$ J. T. Bahns, A. Imre, V. K. Vlasko-Vlasov, J. Pearson, J. M. Hiller, L. H. Chen, and U. Welp, Appl. Phys. Lett. 91, 081104 (2007). 